\title{
Rapid and Quantitative Detection of Human Antibodies against the 2019 Novel Coronavirus SARS CoV2 and Its Variants as a Result of Vaccination and Infection
}

\author{
Benjamin Taubner, ${ }^{a}$ Ruben Peredo-Wende, ${ }^{b}$ Ananthakrishnan Ramani, ${ }^{c}$ Gurpreet Singh, ${ }^{\text {d }}$ Klemen Strle,, ef (D) Nathaniel C. Cady ${ }^{a}$ \\ aCollege of Nanoscale Science \& Engineering, SUNY Polytechnic Institute, Albany, New York, USA \\ bDivision of Rheumatology, Albany Medical Center, Albany, New York, USA \\ 'Division of Infectious Diseases, Albany Medical Center, Albany, New York, USA \\ dInternal Medicine, Albany Medical Center, Albany, New York, USA \\ eWadsworth Center, New York State Department of Health, Albany, New York, USA \\ fSchool of Public Health, University at Albany, Albany, New York, USA
}

ABSTRACT Measuring the antibody response to 2019 SARS CoV2 is critical for diagnostic purposes, for monitoring the prevalence of infection, and for gauging the efficacy of the worldwide vaccination effort for COVID-19. In this study, a microchipbased grating-coupled fluorescent plasmonic (GC-FP) assay was used to measure antibody levels that resulted from COVID-19 infection and vaccination. In addition, we measured the relative antibody binding toward antigens from the CoV2 virus variants strains B.1.1.7 (Alpha) and B.1.351 (Beta). Antibody levels against multiple antigens within the SARS CoV2 spike protein were significantly elevated for both vaccinated and infected individuals, while those against the nucleocapsid ( $\mathrm{N}$ ) protein were only elevated for infected individuals. GC-FP was effective for monitoring the IgG-based serological response to vaccination throughout the vaccination sequence and also resolved acute (within hours) increases in antibody levels. A significant decrease in antibody binding to antigens from the B.1.351 variant, but not B.1.1.7, was observed for all vaccinated subjects when measured by GC-FP compared to the 2019 SARS CoV2 antigens. These results were corroborated by competitive enzymelinked immunosorbent assay (ELISA). Collectively, the findings suggest that GC-FP is a viable, rapid, and accurate method for measuring both overall antibody levels to SARS CoV2 and relative antibody binding to viral variants during infection or vaccination.

IMPORTANCE In this work, a novel biosensor technology was used to measure antibody levels that resulted from vaccination against COVID-19 and/or from infection with the virus. Importantly, this approach enables quantification of antibody levels, which can provide information about the timing and level of immune response. Due the multiplexed nature of this approach, antibody binding to both the original 2019 SARS CoV-2 strain and variant strains can be performed simultaneously and in a short (30-min) time frame.

KEYWORDS COVID-19, CoV2, antibody, biosensor, plasmonic, quantitative, multiplex, vaccine, variant, diagnostic, detection

T: he 2019 novel coronavirus, severe acute respiratory syndrome/coronavirus disease

2 (SARS CoV2/COVID-19) has resulted in millions of deaths worldwide and has spurred the development of novel diagnostic strategies, detection technologies, and vaccination approaches. Monitoring an individual's antibody responses to CoV2 antigens has become paramount from an epidemiological perspective, but also as a means
Citation Taubner B, Peredo-Wende R, Raman A, Singh G, Strle K, Cady NC. 2021. Rapid and quantitative detection of human antibodies against the 2019 novel coronavirus SARS CoV2 and its variants as a result of vaccination and infection. Microbiol Spectr 9:e00890-21. https://doi.org/10.1128/Spectrum.00890-21 Editor Tulip Jhaveri, University of Mississippi Medical Center

Copyright $\odot 2021$ Taubner et al. This is an open-access article distributed under the terms of the Creative Commons Attribution 4.0 International license.

Address correspondence to Nathaniel C. Cady, ncady@sunypoly.edu.

Received 13 July 2021

Accepted 27 August 2021

Published 29 September 2021 
of determining the efficacy of vaccination. By measuring the levels of antibodies (primarily lgG) in human blood or other bodily fluids, an individual's prior infection history, as well as their serological response to vaccination, can be elucidated. Monitoring the stability and/or decline of antibody levels over time is important in estimating how long individuals will retain immunity (1).

Beyond assessing human serological response to infection or vaccination, it is important to understand how an individual's immune system will respond to a growing number of novel CoV2 variants. A core area of concern is mutations in the spike protein, which could interfere with antibody binding and subsequently affect the blockade (neutralization) of viral entry into human cells via the ACE2 receptor (2). This could ultimately result in breakthrough infections for individuals who were previously infected or vaccinated (2). Recent studies have shown that emerging variants in the United Kingdom (B.1.1.7/Alpha variant) and South Africa (B.1.351/Beta variant) are not as effectively neutralized by blood serum from vaccinated individuals, nor from those who were previously infected with the original 2019 SARS CoV2 strain (2-4). This is also an area of concern for variants emerging in other regions, including Brazil and India (5, 6). This highlights the need for sensitive, specific, high-throughput assays to monitor antibody levels and predict effectiveness.

Multiplexed quantitative serological assays, which allow both the determination of antibody levels in response to infection and vaccination and the ability to assess relative antibody-neutralizing capacity, provide an attractive diagnostic solution. Established methods of assessing antibody response to SARS CoV2 and its variants include cell-based viral neutralization assays $(2,3,7,8)$ and competitive in vitro binding assays such as enzyme-linked immunosorbent assay (ELISA) $(9,10)$. Previously we demonstrated a grating-coupled fluorescent plasmonic (GC-FP) biosensor platform for rapid (30 min), quantitative, multiplexed detection of human antibody response to both Lyme disease and COVID-19 infection (11, 12). The GC-FP detection ratio (ratio of antibody binding to target antigens versus negative-control proteins) for human serum and dried blood spot samples correlated strongly with standard antibody testing approaches, including microsphere immunoassay (MIA) and ELISA (12). Notably, we found that dried blood spot samples as well as more traditional blood serum samples yielded high sensitivity and selectivity for diagnosing prior COVID-19 infection.

In the study presented here, we modified GC-FP detection microchips to include additional antigens from variant strains of SARS CoV2, including B.1.1.7 and B.1.351. Using these new detection microchips, we were able to simultaneously monitor antibody levels against multiple 2019 SARS CoV2 antigens for individuals throughout the vaccination sequence and for all three vaccine types currently approved for use in the United States -Pfizer-BioNTech (13), Moderna (14), and single-dose Johnson \& Johnson (15). We also assessed the relative binding of antibodies to original and variant strains of SARS CoV2 antigens for multiple exposure scenarios, including (i) acute severe (hospitalized) infection, (ii) mild infections that did not lead to hospitalization, (iii) vaccination with all three vaccines, (iv) and a combination of prior infection and vaccination. Our results demonstrate that GC-FP is an effective and sensitive method to monitor antibody levels in response to vaccination and can determine relative antibody binding levels to original and variant 2019 SARS CoV2 antigens, all in a single, rapid test.

\section{RESULTS}

Determination of human antibody response to 2019 SARS CoV2 vaccination. Dried blood samples from individuals with no known previous COVID-19 infection ( $n=52$ ) and individuals 2 weeks past full vaccination with either Pfizer-BioNTech ( $n=17)$, Moderna $(n=8)$, or Johnson \& Johnson $(n=9)$ vaccines were tested using the GC-FP assay to determine antibody levels against three SARS CoV2 spike protein antigens (the S1 fragment [S1], the extracellular domain [S1S2], and the receptor binding domain $[R B D]$ of the spike protein) and the nucleocapsid protein (N). As we described in previous work (12), the GC-FP diagnostic ratio provides a quantitative measure of 
A

S1 - 2019 CoV2

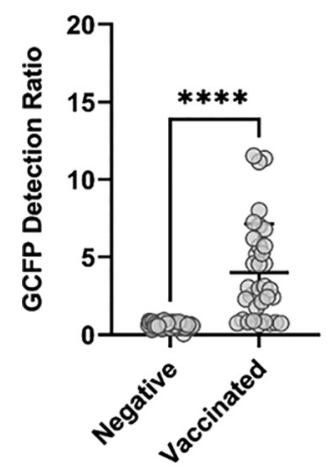

RBD - 2019 CoV2

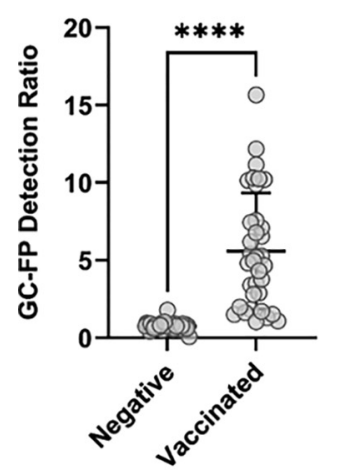

S1S2 - 2019 CoV2

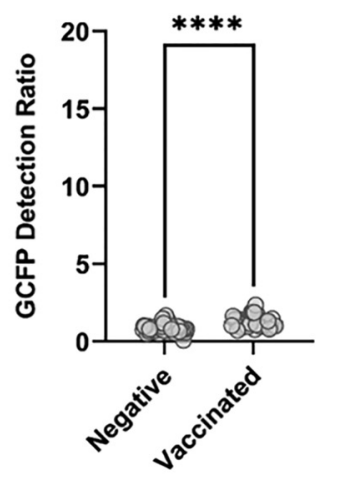

N - 2019 CoV2

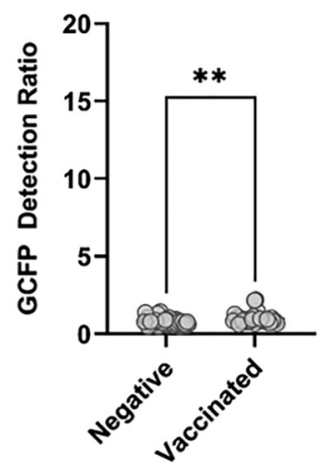

B

S1 - 2019 CoV2

S1S2 - 2019 CoV2
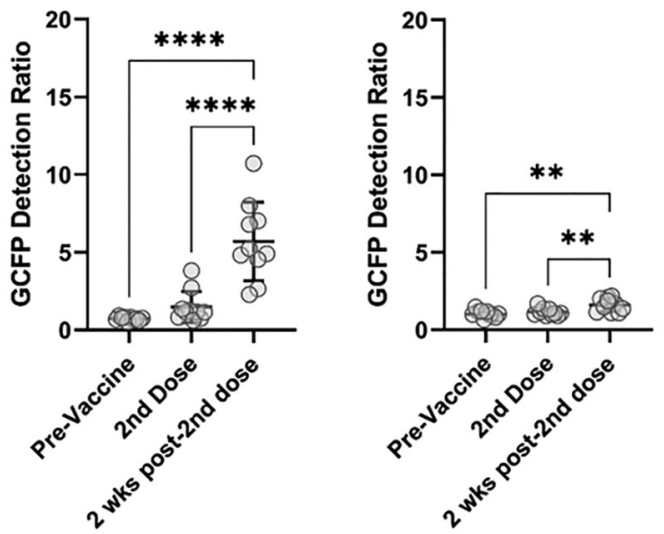

RBD - 2019 CoV2

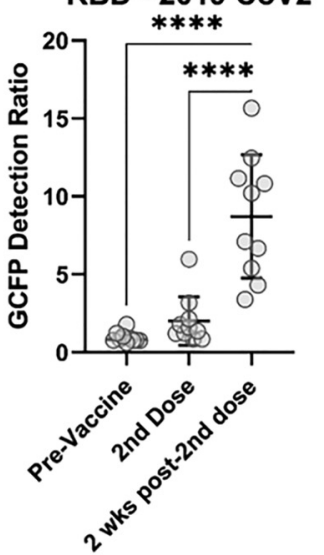

N - 2019 Cov2

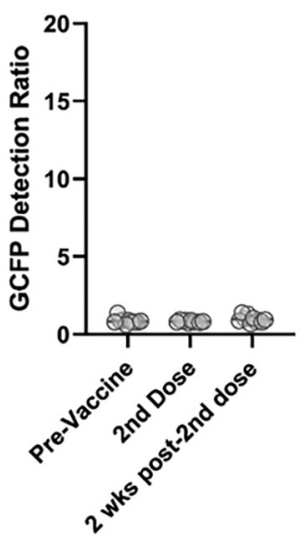

FIG 1 (A) Human IgG levels against SARS CoV2 antigens, measured by GC-FP, for vaccinated individuals versus unvaccinated individuals. The Mann-Whitney test was used to determine statistical significance. (B) Human IgG levels against SARS CoV2 antigens throughout the vaccination sequence (Pfizer-BioNTech) for 10 subjects, collected prevaccination, at the time of the 2nd dose of vaccine, and 2 weeks after the 2nd dose of vaccine. One-way analysis of variance (ANOVA) followed by Dunnett's multiple-comparison testing was performed $\left({ }^{*}, P=0.03 ;{ }^{* *}, P=0.002 ;{ }^{* * *}, P=0.0002 ;{ }^{* * *}, P<0.0001\right)$.

antibody levels and correlates well with established serological techniques such as MIA and ELISA. In the current work, antibody levels in vaccinated individuals were significantly elevated for all spike antigens (Mann-Whitney, $P<0.0001$ ), with the highest mean increase in antibody levels observed for the S1 and RBD antigens (Fig. 1A). A nominal increase in antibody levels was also observed against the S1S2 antigen and the $\mathrm{N}$ protein, but reactivity to these antigens was significantly less than for $\mathrm{S} 1$ and RBD. Receiver operator characteristic (ROC) analysis on these data (see Fig. S2 in the supplemental material) showed that antibody levels against S1 were diagnostic for vaccination status with $61 \%$ sensitivity and $100 \%$ specificity, while levels against RBD were diagnostic with $80 \%$ sensitivity and $100 \%$ specificity. Antibody levels for S1S2 and $\mathrm{N}$ resulted in low area under the curve (AUC) results from the ROC analysis and were considered insufficient for diagnostic purposes. Because all three vaccines should only elicit immunological response to the SARS CoV2 spike antigen, the lack of a diagnostic antibody response for the $\mathrm{N}$ protein was expected.

Dried blood samples were also tested using GC-FP for 10 individuals throughout vaccination with the Pfizer-BioNTech vaccine (prevaccine, at the time of the 2 nd dose, and 2 weeks after 2 nd dose). Antibody levels (as determined by GC-FP detection ratio) were significantly higher for the S1, S1S2, and RBD antigens at both the time of the 2nd dose and 2 weeks after the second dose (Fig. 1). As expected, antibodies against 


\section{IgG Levels through Vaccination Sequence (Pfizer)}

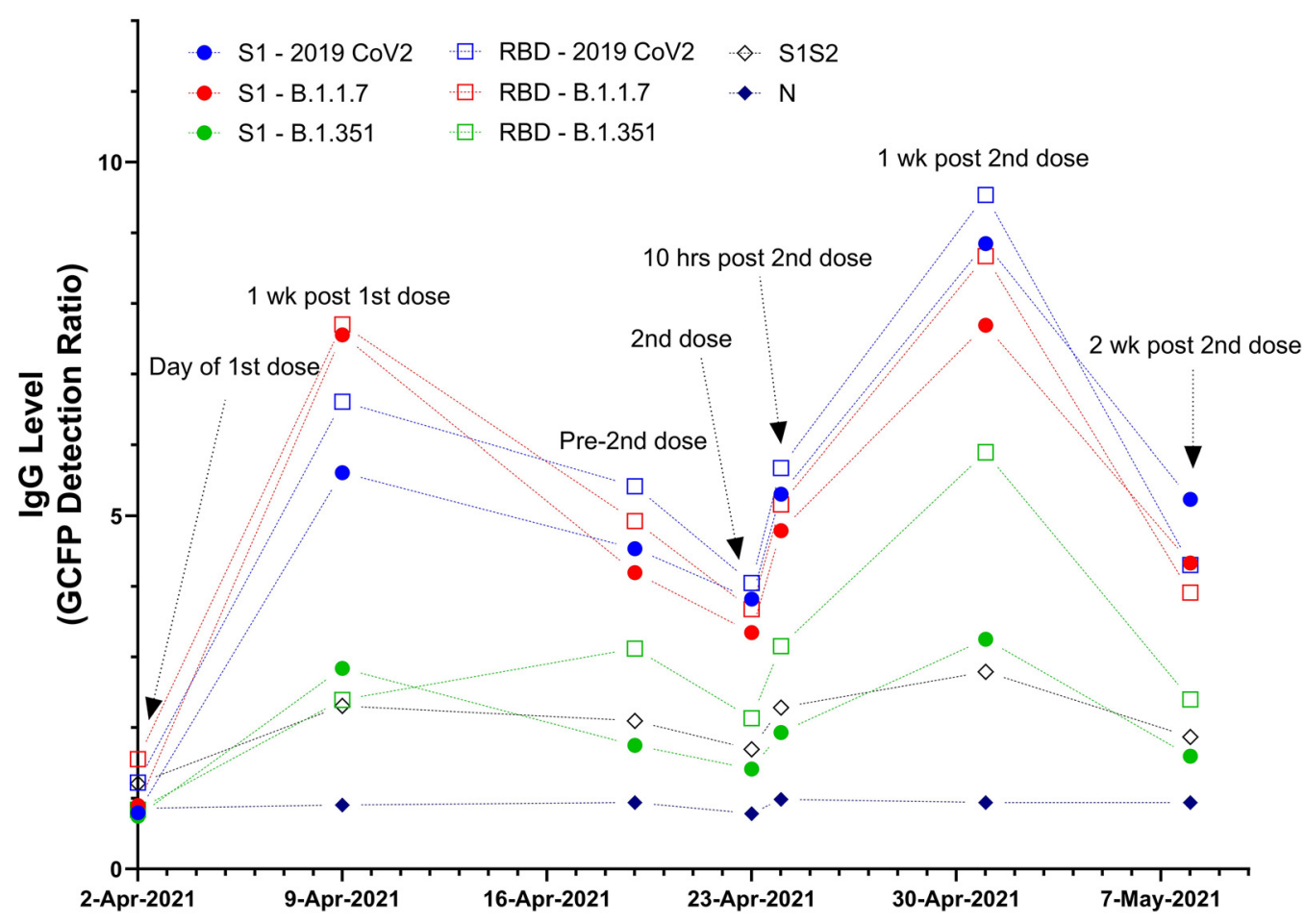

FIG 2 Human IgG levels for a single individual over the course of vaccination with the Pfizer-BioNTech vaccine, measured with GC-FP.

the $\mathrm{N}$ antigen were not detected at any of these time points, since all three vaccines utilize the spike antigen (and not the $\mathrm{N}$ antigen) to induce immune response.

GC-FP was also used to detect antibody levels against SARS CoV2 antigens and antigens from variants B.1.1.7 and B.1.351 at additional time points throughout the vaccination sequence (Pfizer-BioNTech) for an individual subject (Fig. 2). Increased antibody levels were observed against spike antigens (S1, S1S2, and RBD) within 1 week after the 1st dose. Antibody levels then declined slightly until the 2nd dose, when they increased at both $10 \mathrm{~h}$ and 1 week post-2nd dose. Finally, levels declined at 2 weeks post-2nd dose. The changes in antibody levels correlate well with what is expected during the vaccination sequence, wherein antibody levels should increase after each dose but then decline to a stable level over time. Antibody responses to S1 or RBD from the B.1.1.7 variant were similar to responses to antigens from the original 2019 SARS CoV2 strain, but these responses were dramatically reduced for the RBD and S1 antigens from the B.1.351 variant throughout the vaccination course. These data illustrate the potential to quantitatively measure antibody response to vaccination with high resolution (through time).

Determination of human antibody binding to 2019 SARS CoV2 antigens and its variants. GC-FP testing was performed on serum from acutely infected (hospitalized) individuals and dried blood samples from individuals who were (i) uninfected/prevaccine (had no known prior infection with COVID-19 and were not vaccinated), (ii) hospitalized (infected with COVID-19 and hospitalized due to infection), (iii) nonhospitalized (were infected with COVID-19 and at least 4 weeks postrecovery), (iv) vaccinated (Pfizer, Moderna, J\&J; were at least 2 weeks past the final dose of the Pfizer-BioNTech, Moderna, or Johnson \& Johnson vaccine), or (v) CoV2 positive and vaccinated (previously infected with COVID-19 and then fully vaccinated with the Pfizer-BioNTech or Moderna vaccine). The results of this study are shown in Fig. 3. Compared to uninfected/unvaccinated individuals, vaccinated individuals (with and without prior infection) had significantly higher antibody levels against S1 and RBD antigens. Acutely infected (hospitalized) individuals had elevated antibody levels for S1S2 and N 
S1 - 2019 CoV2

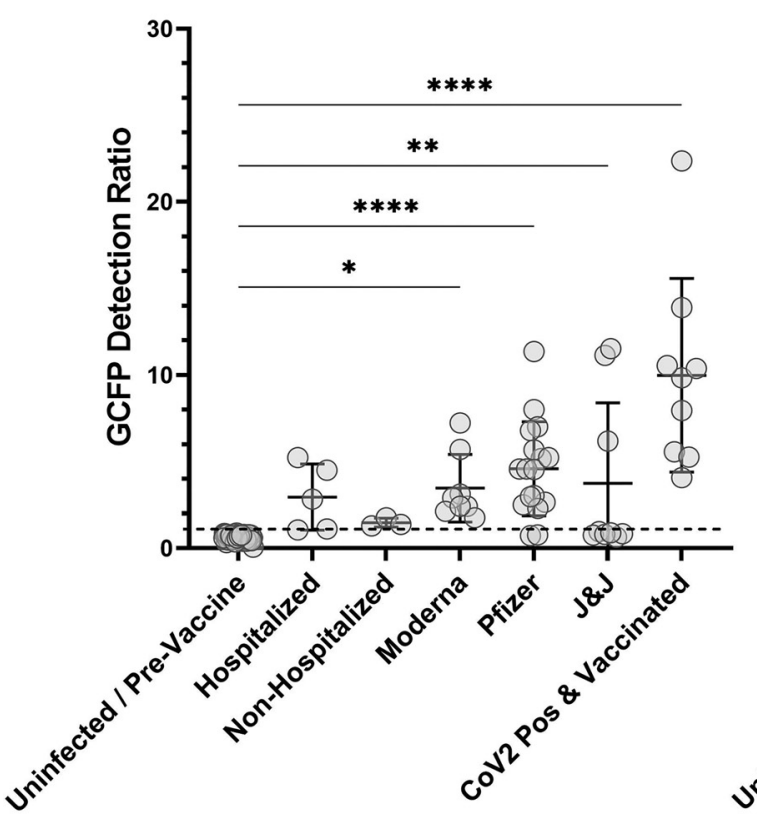

RBD - 2019 CoV2

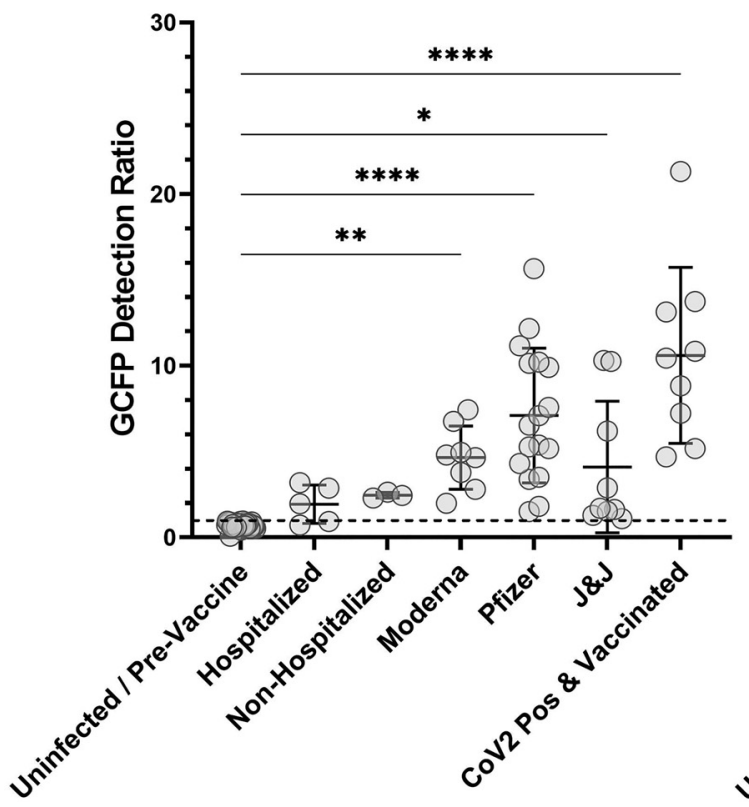

S1S2 - 2019 CoV2

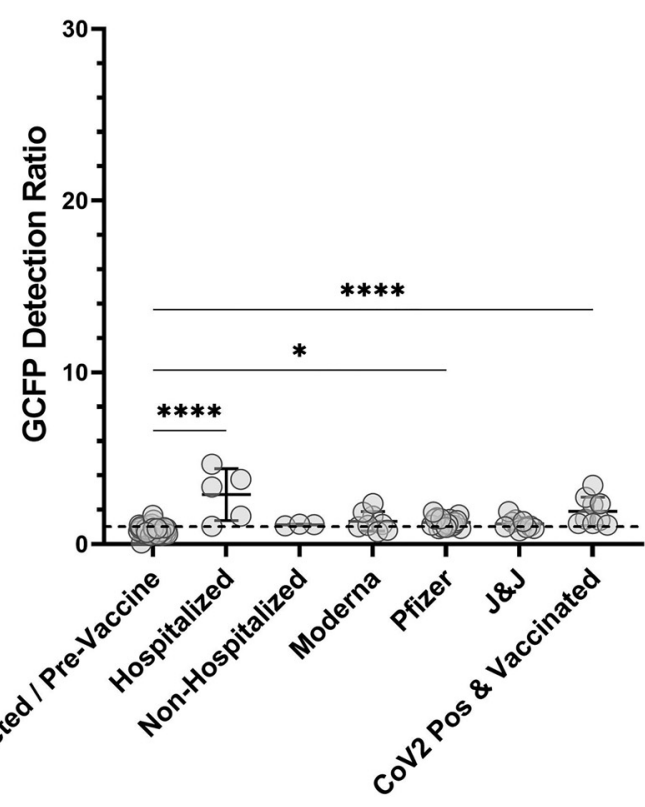

N - 2019 CoV2

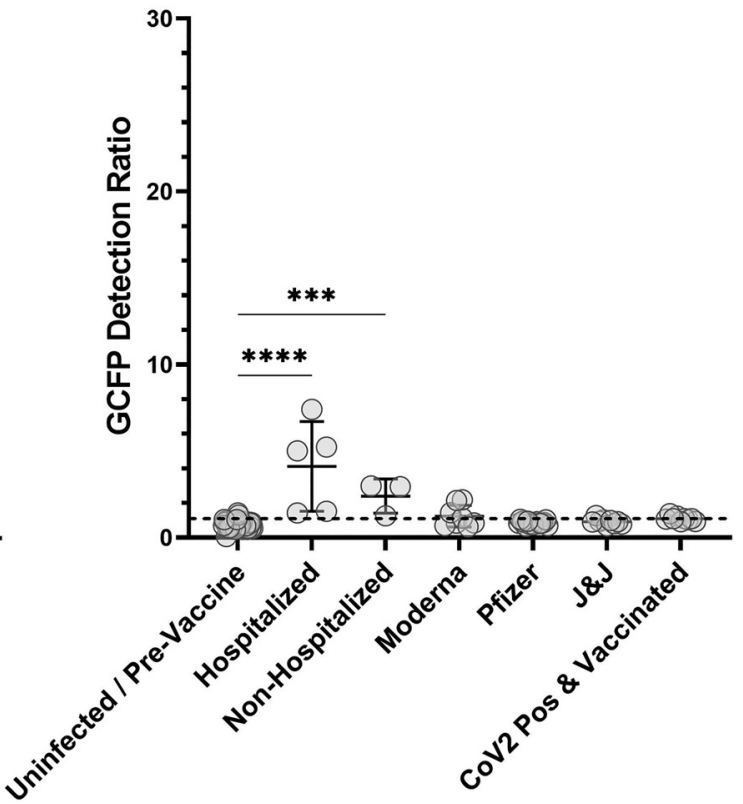

FIG 3 lgG levels against SARS CoV2 antigens for uninfected, previously infected, and vaccinated individuals. Uninfected samples were collected prior to vaccination, from individuals who reported no prior COVID-19 symptoms, and tested negative via PCR and/or antibody testing $(n=42)$. Other samples were from PCR-confirmed COVID-19-positive subjects who were hospitalized $(n=3)$, PCR-confirmed COVID-19-positive subjects who were not hospitalized CoV2 $(n=5)$, and previously COVID-19-positive subjects who received subsequent vaccination $(n=9)$. Samples were also collected from subjects who were at least 2 weeks past full vaccination with Pfizer-BioNTech $(n=17)$ or Moderna $(n=8)$ or 2 weeks past receiving the Johnson \& Johnson vaccine $(n=9)$. One-way ANOVA followed by Dunnett's multiple-comparison test was performed $\left({ }^{*}, P=0.03 ;{ }^{* *}, P=0.002 ;{ }^{* *}\right.$, $\left.P=0.0002 ;{ }^{* * * *}, P<0.0001\right)$.

antigens, while nonhospitalized patients showed increased antibody levels only against N. Notably, individuals who were vaccinated after prior infection had the highest mean antibody levels against S1 and RBD. This is consistent with the fact that these individuals were effectively exposed to the spike antigen at three different time points (during infection and at the 1 st and 2 nd doses of vaccine). 

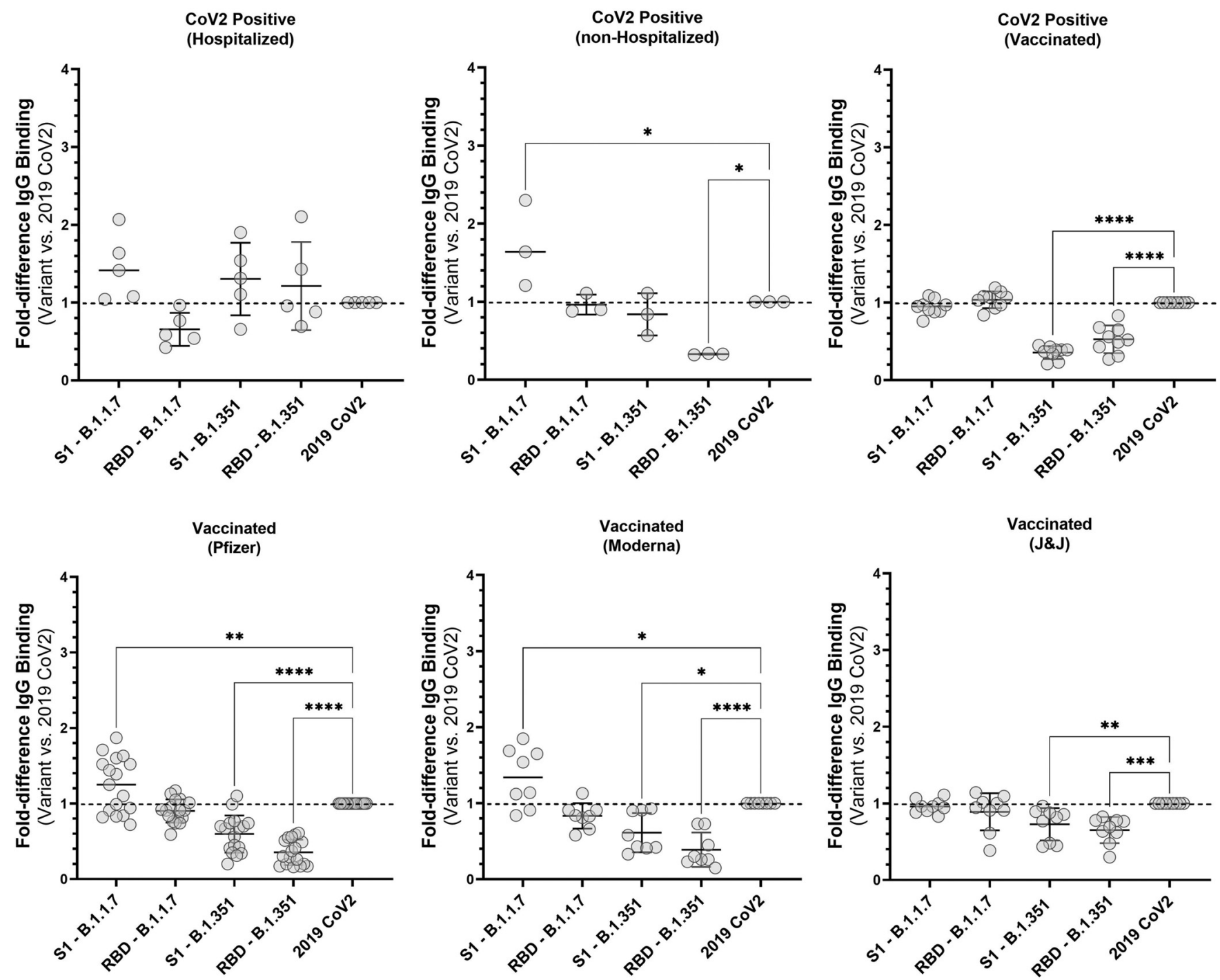

FIG 4 Fold-difference in antibody levels against antigens from SARS CoV2 variant strains B.1.1.7 and B.1.351 versus antigens from the original 2019 SARS CoV2 strain. Samples included those from individuals who were hospitalized $(n=3)$, nonhospitalized CoV2-positive $(n=5)$, previously CoV2-positive with subsequent vaccination $(n=9)$, and at least 2 weeks past vaccination with Pfizer-BioNTech $(n=17)$, Moderna $(n=8)$, or Johnson \& Johnson vaccine $(n=9)$. One-way ANOVA followed by Dunnett's multiple-comparison test was performed $\left(^{*}, P=0.03 ;{ }^{* *}, P=0.002 ;{ }^{* *}, P=0.0002 ;{ }^{* * *}, P<0.0001\right)$.

Between individual subjects, antibody levels were highly variable, regardless of the mode of COVID-19 exposure or vaccination status (Fig. 3 and Fig. S3). Another observation was that antibody response was somewhat dependent upon the manufacturer of the vaccine used; the Pfizer vaccine elicited the highest mean response, whereas the Moderna and $J \& J$ vaccines elicited a lower mean response. We also observed that Nspecific IgG response for CoV2 positive + vaccinated individuals was low/borderline undetectable. This may be because these individuals were vaccinated between 4.5 and 54 weeks postinfection; thus, some individuals may have experienced waning antibody titers from the initial infection. Further, antibody response can be highly variable, especially if the initial infection was weak or asymptomatic.

To elucidate relative antibody binding levels to variant antigens versus 2019 SARS CoV2 antigens, we plotted the fold-difference (binding to variant antigen versus 2019 SARS CoV2 antigen) in antibody levels between variant antigens and the 2019 SARS CoV2 antigens (Fig. 4). For all vaccinated individuals, antibody binding to B.1.351 antigens (both RBD and S1) was reduced compare to that of 2019 SARS CoV2 antigens. Antibody binding to B.1.1.7 antigens was either equivalent to or slightly higher than that of 2019 SARS CoV2 antigens for these same individuals. For previously infected 
individuals (hospitalized and nonhospitalized), there were only minor differences in antibody levels for variant antigens versus 2019 SARS CoV2 antigens $(P>0.03$ or not significant). Information regarding the specific strain that these individuals were infected with is not available. If these individuals were infected with one of the variant strains, then antibody binding to variant antigens versus 2019 SARS CoV2 antigens is expected to be insignificant.

To determine if these results were due to differences in antibody affinity toward variant versus 2019 SARS CoV2 antigens, we compared the GC-FP results to a competitive ELISA in which blood samples were mixed with ACE2 receptor protein (the cellular target of SARS CoV2) and allowed to competitively bind to 2019 SARS CoV2 RBD antigen or the B.1.1.7 and B.1.351 RBD variants. Competitive ELISA results were similar to GC-FP results (Fig. 5A and B), showing both significantly reduced antibody binding (GC-FP) and reduced binding inhibition (competitive ELISA) for the B.1.351 RBD variant. When quantitative dilution testing was performed for a single Pfizer-BioNTech-vaccinated individual and a single acutely infected (hospitalized) individual, significant differences in binding inhibition were observed for both B.1.1.7 and B.1.351 RBD variants (Fig. 5C and D). When the fold difference in antibody binding (GC-FP) and the percentage of binding inhibition (competitive ELISA) were compared, there was close correlation between the two methods (Fig. 5E and F and Fig. S4). The values plotted in Fig. 5E and $\mathrm{F}$ were shown to be correlated (Pearson $r=0.89, P=0.02$; Fig. S4), suggesting that GC-FP has utility for assessing relative antibody binding levels and/or avidity to antigens from 2019 SARS CoV2 and variant strains of the virus.

\section{DISCUSSION}

We previously demonstrated that GC-FP is a rapid and accurate technique for detection of antibodies that result from COVID-19 infection (12). In the current study, we extended this work to show that GC-FP can measure the level of antibodies resulting from vaccination and that it can quantitatively measure the increase in antibody levels during the course of vaccination for multiple target antigens. Using ROC analysis, GC-FP diagnostic ratio thresholds were established to yield a clear cutoff for determining whether or not an individual has been vaccinated. When using the RBD antigen, this results in $80 \%$ sensitivity and $100 \%$ specificity for all three of the currently approved vaccines (Pfizer-BioNTech, Moderna, and Johnson \& Johnson). Furthermore, our results show that antibody levels can be measured with high resolution throughout the course of vaccination, making it a useful tool to track the progression of an individual's serological response to a vaccine. Highlighting the sensitivity of the GC-FP approach, we detected increasing levels of antibodies within just $10 \mathrm{~h}$ of an individual's second dose of the Pfizer-BioNTech vaccine (Fig. 2).

Beyond determination of vaccination status and serological response to vaccination, the multiplexed nature of GC-FP makes it highly amenable to measuring antibody binding to multiple antigens from both the original 2019 SARS CoV2 virus and the emerging variants of this virus. This is extremely important, as variants of the virus continue to emerge $(5,6)$. For example, GC-FP results indicate reduced antibody binding to the RBD and S1 antigens of the B.1.351 (Beta) variant, which was further confirmed through competitive ELISA-based testing (Fig. 5). When individual samples were evaluated by GC-FP and competitive ELISA, reduced antibody binding to antigens from both the B.1.1.7 (Alpha) and B.1.351 variants was observed.

The results of the present study, together with our previous demonstration of GC-FP for COVID-19 antibody detection (12) compare favorably to similar studies using multiplexed, array-based techniques. Using a plasmonic-based approach, Liu et al. demonstrated high-throughput detection of COVID-19 antibodies from human serum and saliva (16). This approach requires significantly more time than GC-FP ( $\sim 2 \mathrm{~h}$ versus $30 \mathrm{~min}$ ), but it has the advantage of measuring the relative avidity of antibodies toward target antigens. In another study (17), researchers demonstrated an elegant, high-throughput microfluidic approach to COVID-19 antibody detection. While this approach enables 
A

ACE2 Competitive Binding Assay (Vaccinated Subjects)

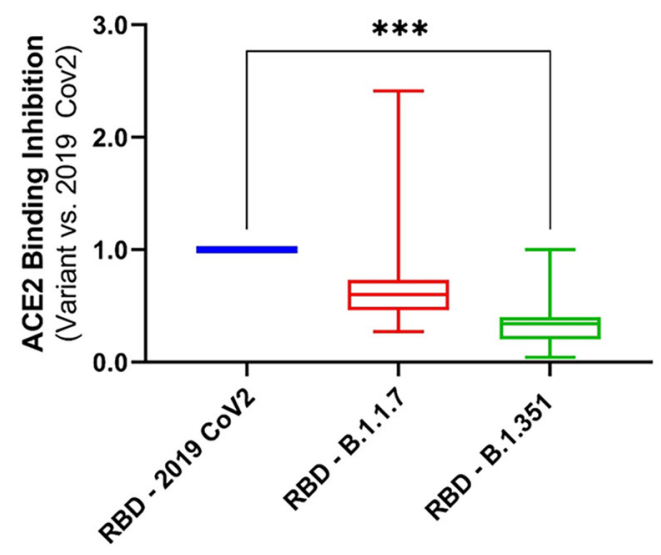

C

Vaccinated (Pfizer)

(Dried Blood Spot)

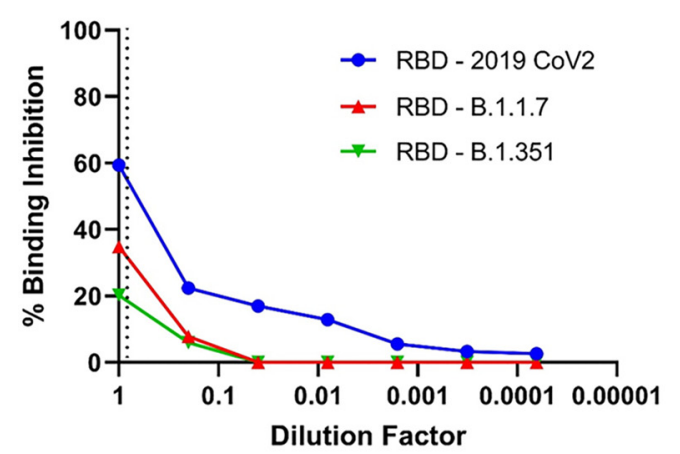

E

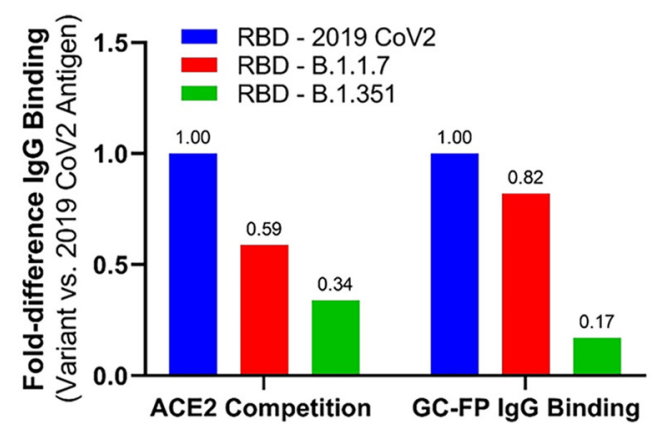

B

GC-FP

(Vaccinated Subjects)

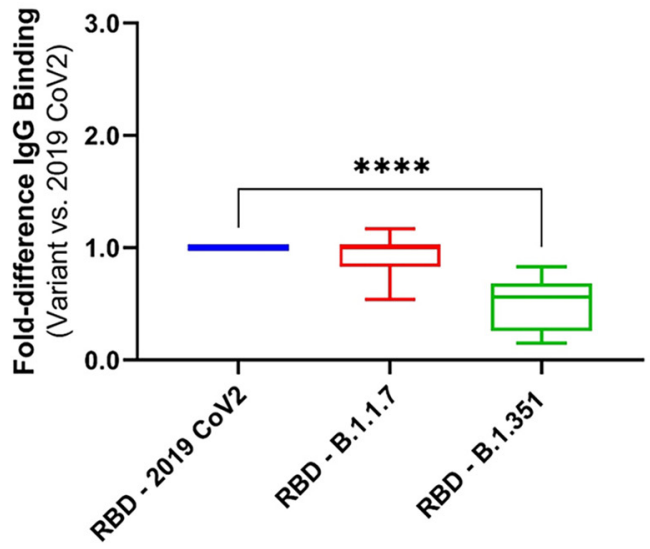

D CoV2 Positive (Hospitalized)

(Serum)

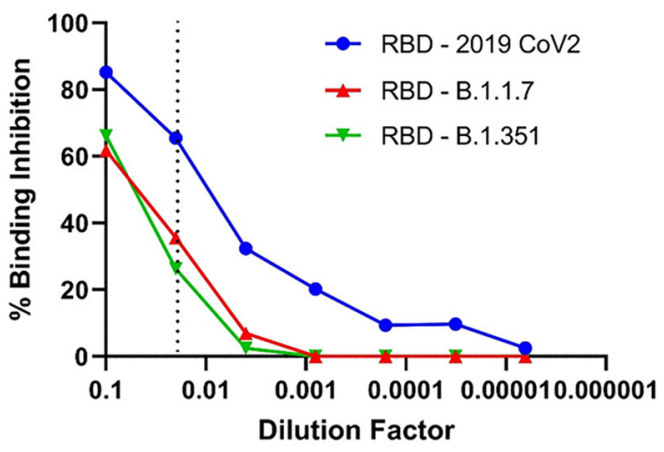

F

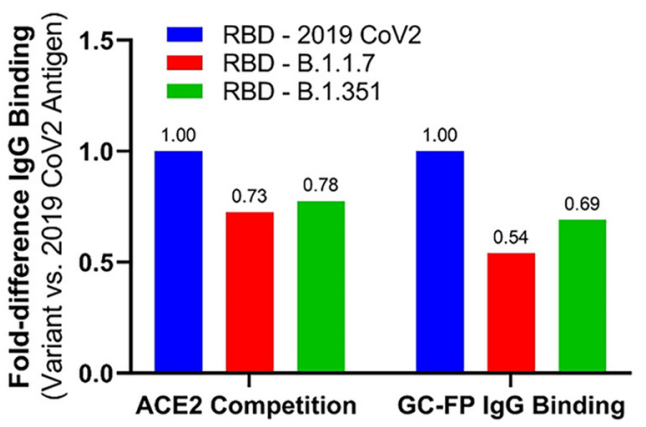

FIG 5 (A and B) IgG levels from dried blood spots measured by GC-FP diagnostic ratio compared to competitive ELISA by eluate from the same dried blood spot samples. Both GC-FP and ACE2 competitive binding were performed for RBD antigen from the original 2019 SARS CoV2 and the variant strains B.1.1.7 and B.1.351. Testing was performed with dried blood spots collected from vaccinated subjects (3 Pfizer-BioNTech, 3 Moderna, 2 Johnson \& Johnson) and subjects who were both previously infected and then vaccinated with Pfizer-BioNTech or Moderna vaccines $(n=3)$. One-way ANOVA followed by Dunnett's multiple-comparison test was performed $\left({ }^{*}, P=0.03 ;{ }^{* *}, P=0.002 ;{ }^{* *}\right.$, $P=0.0002{ }^{* * * *}, P<0.0001$ ). (C and D) The percentage of ACE2 binding inhibition from the competitive ELISA is shown for a dried blood spot sample (C) from a Pfizer-BioNTech-vaccinated subject and blood serum from a hospitalized, COVID-positive subject (D). Vertical dotted lines represent the dilution factor used in the corresponding GC-FP test for each sample. (E and F) The fold difference in binding inhibition and fold difference in GC-FP diagnostic ratio were plotted for variant antigens (RBD B.1.1.7 and RBD B.1.351) versus RBD 2019 CoV2. 
TABLE 1 Proteins and peptides used for generating GC-FP detection microchips

\begin{tabular}{ll}
\hline Antigen/protein & Source \\
\hline Human IgG (positive control) & Thermo Fisher \\
Human serum albumin/HSA (negative control) & Sino Biological \\
2019 SARS CoV2 nucleocapsid (N) & Sino Biological \\
2019 SARS CoV2 RBD (RBD) & Sino Biological \\
2019 SARS CoV2 S1S2 + extracellular domain/ECD (S1S2) & Sino Biological \\
2019 SARS CoV2 S1 (S1) & Sino Biological \\
SARS CoV2 RBD-B.1.1.7/Alpha (N501Y) & Sino Biological \\
SARS CoV2 RBD-B.1.351/Beta (K417N, E484K, N501Y) & Sino Biological \\
SARS CoV2 S1-B.1.351/Beta (K417N, E484K, N501Y, D614G) & Sino Biological \\
SARS CoV2 S1-B.1.1.7/Alpha ( $(\Delta \mathrm{HV69-70, \Delta Y144,} \mathrm{N501Y,} \mathrm{A570D,} \mathrm{D614G,} \mathrm{P681H)}$ & Sino Biological \\
\hline
\end{tabular}

extremely high throughput, it is limited in the number of target antigens that can be implemented and requires complicated printing of human blood samples to prepare detection chips. Further, we previously demonstrated that the GC-FP assay can be used to detect other antibody isotypes (IgA, $\lg M)$ beyond $\lg$, providing even more utility toward sample analysis (12).

In the work presented here, we have demonstrated that GC-FP-based, multiplexed detection of antibody binding from human blood is a useful tool for determining an individual's response to vaccination and the relative binding of antibodies to variants of the 2019 SARS CoV2 virus. When considering the rapid time to result (30 $\mathrm{min}$ ) for the GC-FP assay and the ability to target a large number of antigens at once, this represents a powerful tool for continued management of the global COVID-19 pandemic, with broad applicability to other diseases and vaccines.

\section{MATERIALS AND METHODS}

Materials. Nucleocapsid protein (N), the S1 fragment of the spike protein (S1), the extracellular domain of the spike protein (S1S2), the receptor binding domain of the spike protein for the 2019 SARS CoV2 virus, S1 variant antigens, RBD variant antigens, and human serum albumin (HSA) were all obtained from Sino Biological, Inc. (Table 1). Positive-control protein, human IgG protein (Hum IgG), SuperBlock blocking buffer, and phosphate-buffered saline (PBS) were obtained from Thermo Fisher Scientific. PBS-Tween (PBS-T) solution consisting of PBS plus $0.05 \%$ (vol/vol) Tween 20 (Sigma-Aldrich) was prepared daily for all experiments. Alexa Fluor 647 labeled anti-human IgG (heavy and light chain) was obtained from Invitrogen/Thermo Fisher Scientific. ACE2 competitive ELISA testing was performed using COVID-19 ACE2 testing kits from RayBiotech (COVID-19 spike variant-ACE2 binding assay kit).

Grating-coupled fluorescent plasmonic biosensor chip preparation. Gold-coated GC-FP biosensor chips were fabricated as described in our previous work $(11,12,18)$. GC-FP chips were also printed as described previously (12). A map of the protein/antigen spots is shown in Fig. S1.

Biological samples. Serum samples were collected from COVID-19 patients admitted to Albany Medical Center from October to December 2020 who enrolled in the study "Defining Genetic and Immune Factors in COVID-19 Severity." All patients had a positive RT-PCR test for CoV2 and were hospitalized due to the severity of their illness. Sera were processed on the day of collection and placed in a $-80^{\circ} \mathrm{C}$ freezer until analyses. The study was approved by the institutional review board (IRB) at Albany Medical Center (protocol 5929).

Dried blood samples were collected using the finger stick method. Lancet devices (27 gauge [ga.]) and Whatman 903 protein saver collection cards were sent to volunteers with instructions and a consent form approved by the State University of New York (SUNY) Polytechnic Institute Institutional Review Board (protocolsIRB-2020-10 and IRB-2021-2). Blood droplets were collected, allowed to dry, and then either hand delivered or mailed (via the U.S. Postal Service) to SUNY Polytechnic Institute. Following receipt of dried blood spot (DBS) samples, a 6-mm diameter biopsy punch was used to remove the samples from the collection cards, which were then soaked in $500 \mu \mathrm{l}$ of PBS-T for $\sim 12 \mathrm{~h}$ at $4^{\circ} \mathrm{C}$ with rocking. Samples were collected from (i) participants who had no known exposure to COVID-19 and/or tested negative for COVID-19 infection, (ii) vaccinated individuals at different time points throughout the vaccination sequence, including prevaccination, at the second dose, and 2 weeks after the second dose, (iii) vaccinated individuals at a single time point, a minimum of 2 weeks after the final vaccination dose, and (iv) vaccinated individuals who were previously diagnosed with COVID-19 infection via RT-PCR. The age, gender, and exposure/vaccination information for all participants represented in this work are listed in Tables S1 and S2.

GC-FP detection assay and ACE2 competitive assay. GC-FP microchips were processed using the conditions described previously (12). The total assay time from sample introduction to chip imaging was $30 \mathrm{~min}$. For serum testing, a standard dilution of serum in PBS-T (1:50) was used. For dried blood spot 
testing, undiluted extract (from extraction in $500 \mu \mathrm{l} \mathrm{PBS-T)} \mathrm{from} \mathrm{the} \mathrm{6-mm-diameter} \mathrm{segment} \mathrm{of} \mathrm{the}$ blood collection card was used in place of serum. Ciencia image analysis LabView software was used to define a region of interest (ROI) for each individual spot on the GC-FP biosensor chip, and the fluorescence intensity of each spot was measured. The fluorescence intensity of all spots was normalized to the human $\lg \mathrm{G}$ (Hum lgG) internal-control spots on each chip to account for variability between individual chips and individual experiments, generating a GC-FP detection ratio for every protein/antigen included in the GC-FP microchip (12):

$$
\text { GC-FP Detection Ratio }=\frac{\bar{x} \text { target spot intensity }}{(\bar{x} \text { neg. ctrl. spot intensity })+(3 \sigma \text { neg. ctrl. spot intensity })}
$$

To determine if GC-FP antibody binding data for 2019 SARS CoV2 versus that for B.1.1.7 and B.1.351 variant antigens was consistent with standard methods, an ELISA-based ACE2 competitive binding assay (Ray Biotech) was used, as per the manufacturer's instructions. Additional details are provided in the supplemental material. The percentage of binding inhibition was calculated for each sample with the following method:

$$
\% \text { Binding Inhibition }=1-\left(\frac{\bar{x} \text { sample absorbance at } 450 \mathrm{~nm}}{(\bar{x} \text { positive control absorbance at } 450 \mathrm{~nm})}\right)
$$

Data analysis. GC-FP diagnostic ratio data and percentage binding inhibition data for the ACE2 competitive assay were analyzed using GraphPad Prism 8.0 software (ROC analysis, correlation, and statistical analysis).

\section{SUPPLEMENTAL MATERIAL}

Supplemental material is available online only.

SUPPLEMENTAL FILE 1, PDF file, $1 \mathrm{MB}$.

\section{REFERENCES}

1. Chia WN, Zhu F, Ong SWX, Young BE, Fong SW, Le Bert N, Tan CW, Tiu C, Zhang J, Tan SY, Pada S, Chan YH, Tham CYL, Kunasegaran K, Chen MI, Low JGH, Leo YS, Renia L, Bertoletti A, Ng LFP, Lye DC, Wang LF. 2021. Dynamics of SARS-CoV-2 neutralising antibody responses and duration of immunity: a longitudinal study. Lancet Microbe 2:E240-E249. https://doi .org/10.1016/S2666-5247(21)00025-2.

2. Hoffmann M, Arora $P, G r o ß ~ R$, Seidel A, Hörnich BF, Hahn AS, Krüger N, Graichen L, Hofmann-Winkler H, Kempf A, Winkler MS, Schulz S, Jäck HM, Jahrsdörfer B, Schrezenmeier H, Müller M, Kleger A, Münch J, Pöhlmann S. 2021. SARS-CoV-2 variants B.1.351 and P.1 escape from neutralizing antibodies. Cell 184:2384-2393.e12. https://doi.org/10.1016/j.cell.2021.03.036.

3. Xie X, Liu Y, Liu J, Zhang X, Zou J, Fontes-Garfias CR, Xia H, Swanson KA, Cutler M, Cooper D, Menachery VD, Weaver SC, Dormitzer PR, Shi PY. 2021. Neutralization of SARS-CoV-2 spike 69/70 deletion, E484K and N501Y variants by BNT162b2 vaccine-elicited sera. Nat Med 27:620-621. https://doi.org/10.1038/s41591-021-01270-4.

4. Chang X, Augusto GS, Liu X, Kündig TM, Vogel M, Mohsen MO, Bachmann MF. 2021. BNT162b2 mRNA COVID-19 vaccine induces antibodies of broader cross-reactivity than natural infection but recognition of mutant viruses is up to 10-fold reduced. bioRxiv. https://doi.org/10.1101/2021.03 .13.435222:2021.03.13.435222.

5. Mahase E. 2021. Covid-19: what new variants are emerging and how are they being investigated? BMJ 372:n158. https://doi.org/10.1136/bmj.n158.

6. Wise J. 2021. Covid-19: UK cases of variant from India rise by $160 \%$ in a week. BMJ 373:n1315. https://doi.org/10.1136/bmj.n1315.

7. Muruato $A E$, Fontes-Garfias $C R$, Ren $P$, Garcia-Blanco MA, Menachery VD, Xie X, Shi PY. 2020. A high-throughput neutralizing antibody assay for COVID-19 diagnosis and vaccine evaluation. Nat Commun 11:4059. https://doi.org/10.1038/s41467-020-17892-0.

8. Tan CW, Chia WN, Qin X, Liu P, Chen MI, Tiu C, Hu Z, Chen VC, Young BE, Sia WR, Tan YJ, Foo R, Yi Y, Lye DC, Anderson DE, Wang LF. 2020. A SARSCoV-2 surrogate virus neutralization test based on antibody-mediated blockage of ACE2-spike protein-protein interaction. Nat Biotechnol 38: 1073-1078. https://doi.org/10.1038/s41587-020-0631-z.

9. Valdivia A, Torres I, Latorre V, Francés-Gómez C, Albert E, Gozalbo-Rovira R, Alcaraz MJ, Buesa J, Rodríguez-Díaz J, Geller R, Navarro D. 2021. Inference of SARS-CoV-2 spike-binding neutralizing antibody titers in sera from hospitalized COVID-19 patients by using commercial enzyme and chemiluminescent immunoassays. Eur J Clin Microbiol Infect Dis 40: 485-494. https://doi.org/10.1007/s10096-020-04128-8.
10. Peterhoff $D$, Glück V, Vogel M, Schuster $P$, Schütz A, Neubert $P$, Albert V, Frisch S, Kiessling M, Pervan P, Werner M, Ritter N, Babl L, Deichner M, Hanses F, Lubnow M, Müller T, Lunz D, Hitzenbichler F, Audebert F, Hähnel V, Offner R, Müller M, Schmid S, Burkhardt R, Glück T, Koller M, Niller HH, Graf B, Salzberger B, Wenzel JJ, Jantsch J, Gessner A, Schmidt B, Wagner R. 2021. A highly specific and sensitive serological assay detects SARS-CoV-2 antibody levels in COVID-19 patients that correlate with neutralization. Infection 49:75-82. https://doi.org/10.1007/s15010-020-01503-7.

11. Chou E, Lasek-Nesselquist E, Taubner B, Pilar A, Guignon E, Page W, Lin YP, Cady NC. 2020. A fluorescent plasmonic biochip assay for multiplex screening of diagnostic serum antibody targets in human Lyme disease. PLoS One 15:e0228772. https://doi.org/10.1371/journal.pone.0228772.

12. Cady NC, Tokranova N, Minor A, Nikvand N, Strle K, Lee WT, Page W, Guignon E, Pilar A, Gibson GN. 2021. Multiplexed detection and quantification of human antibody response to COVID-19 infection using a plasmon enhanced biosensor platform. Biosens Bioelectron 171:112679. https://doi.org/10.1016/j.bios.2020.112679.

13. Polack FP, Thomas SJ, Kitchin N, Absalon J, Gurtman A, Lockhart S, Perez JL, Pérez Marc G, Moreira ED, Zerbini C, Bailey R, Swanson KA, Roychoudhury S, Koury K, Li P, Kalina WV, Cooper D, Frenck RW, Hammitt LL, Türeci Ö, Nell H, Schaefer A, Ünal S, Tresnan DB, Mather S, Dormitzer PR, Şahin U, Jansen KU, Gruber WC, C4591001 Clinical Trial Group. 2020. Safety and efficacy of the BNT162b2 mRNA Covid-19 vaccine. N Engl J Med 383:2603-2615. https://doi.org/10.1056/NEJMoa2034577.

14. Baden LR, El Sahly HM, Essink B, Kotloff K, Frey S, Novak R, Diemert D, Spector SA, Rouphael N, Creech CB, McGettigan J, Khetan S, Segall N, Solis J, Brosz A, Fierro C, Schwartz H, Neuzil K, Corey L, Gilbert P, Janes H, Follmann D, Marovich M, Mascola J, Polakowski L, Ledgerwood J, Graham BS, Bennett H, Pajon R, Knightly C, Leav B, Deng W, Zhou H, Han S, Ivarsson M, Miller J, Zaks T, COVE Study Group. 2021. Efficacy and safety of the mRNA-1273 SARS-CoV-2 vaccine. N Engl J Med 384:403-416. https://doi.org/10.1056/NEJMoa2035389.

15. Sadoff J, Gray G, Vandebosch A, Cárdenas V, Shukarev G, Grinsztejn B, Goepfert PA, Truyers C, Fennema H, Spiessens B, Offergeld K, Scheper G, Taylor KL, Robb ML, Treanor J, Barouch DH, Stoddard J, Ryser MF, Marovich MA, Neuzil KM, Corey L, Cauwenberghs N, Tanner T, Hardt K, Ruiz-Guiñazú J, Le Gars M, Schuitemaker H, Van Hoof J, Struyf F, Douoguih M, Group ES, ENSEMBLE Study Group. 2021. Safety and efficacy of single-dose Ad26.COV2.S vaccine against Covid-19. N Engl J Med 384:2187-2201. https://doi.org/10.1056/NEJMoa2101544. 
16. Liu T, Hsiung J, Zhao S, Kost J, Sreedhar D, Hanson CV, Olson K, Keare D, Chang ST, Bliden KP, Gurbel PA, Tantry US, Roche J, Press C, Boggs J, Rodriguez-Soto JP, Montoya JG, Tang M, Dai H. 2020. Quantification of antibody avidities and accurate detection of SARS-CoV-2 antibodies in serum and saliva on plasmonic substrates. Nat Biomed Eng 4:1188-1196. https://doi.org/10.1038/s41551-020-00642-4.

17. Swank Z, Michielin G, Yip HM, Cohen P, Andrey DO, Vuilleumier N, Kaiser L, Eckerle I, Meyer B, Maerkl SJ. 2021. A high-throughput microfluidic nanoimmunoassay for detecting anti-SARS-CoV-2 antibodies in serum or ultralow-volume blood samples. Proc Natl Acad Sci U S A 118:e2025289118. https://doi.org/10.1073/pnas.2025289118.

18. Chou E, Pilar A, Guignon E, Page W, Lin Y-P, Cady N. 2019. Rapid and multiplexed detection of Lyme disease using a grating coupled-fluorescent plasmonics (GC-FP) biosensor platform. Frontiers in biological detection: from nanosensors to systems XI. SPIE BiOS, 2019, San Francisco, CA. https://doi.org/10.1117/12.2507973. 